\title{
Temperature Anomaly in the Tokyo Metropolitan Area during the COVID-19 (coronavirus) Self-Restraint Period
}

\author{
Fumiaki Fujibe \\ Research Center for Climatology, Department of Geography, Tokyo Metropolitan University, Hachioji, Japan
}

\begin{abstract}
Japan underwent a nationwide self-restraint of human activities in spring 2020 to prevent the spread of the COVID-19 infection. In order to evaluate the effect of suppressed human activities on temperature in the Tokyo Metropolitan area, a statistical analysis was made for temperature anomalies during the selfrestraint period using hourly data on the AMeDAS network. The temperature anomaly was defined by the departure from the value that would have been observed without self-restraint, estimated from regression analysis for temperatures at surrounding nonurban stations. It was found that the temperature in central Tokyo (Kitanomaru Park) had a negative anomaly of $-0.49^{\circ} \mathrm{C}$ with a $95 \%$ confidence range of $\pm 0.19^{\circ} \mathrm{C}$ on the average over the strong self-restraint period from April to May. The anomaly was larger in the nighttime than in the daytime, and was found in an area spreading for several tens of kilometers, with a decreasing magnitude according to the distance from Tokyo. These facts indicate a possibility that the reduction of anthropogenic heat release during the self-restraint period resulted in substantial decrease of temperature in the Tokyo Metropolitan area.
\end{abstract}

(Citation: Fujibe, F., 2020: Temperature anomaly in the Tokyo Metropolitan area during the COVID-19 (coronavirus) selfrestraint period. SOLA, 16, 175-179, doi:10.2151/sola.2020-030.)

\section{Introduction}

It is believed that anthropogenic heat release is one of the causes of the urban heat island. A number of numerical simulations using urban canopy parameterization have shown that anthropogenic heat is a leading cause of nighttime urban heat island, apart from complicated interaction of more than one factor (Ichinose et al. 1999; Kusaka and Kimura 2004; Ryu and Baik 2012; Aoyagi et al. 2012; Li et al. 2013). As an observational evidence of the contribution of anthropogenic heat to urban temperature, Fujibe $(1988,2010)$ showed that temperature was lower on holidays (Sundays and national holidays) than on weekdays by about $0.2^{\circ} \mathrm{C}$ in central Tokyo, and $0.1-0.15^{\circ} \mathrm{C}$ in central Osaka, on the basis of statistical analysis of observed data. Earl et al. (2016) reported weekly temperature cycles in Melbourne, and cited a number of references on this theme. The temperature in Beijing has been found to decrease by the order of $0.5^{\circ} \mathrm{C}$ during the Chinese New Year holiday (Zhang et al. 2015; Zhang and Wu 2017; Dou and Miao 2017).

In the spring of 2020, Japan underwent a nation-wide suppression of human activity in response to the Government's request of "self-restraint" to prevent the spread of COVID-19 (coronavirus) infection. The present article describes the temperature anomaly during the self-restraint period in the Tokyo area using data on a routine observation network of the Japan Meteorological Agency (JMA).

Figure 1 shows the geography of the area around Tokyo and distribution of stations, for which details are described in Section 3.1. Tokyo Metropolis has a population of 13.5 million according to the 2015 national census, accounting for $10.6 \%$ of the total population of Japan. Its eastern part is the core area (Tokyo Ward

Corresponding author: Fumiaki Fujibe, Department of Geography, Tokyo Metropolitan University, 1-1 Minami-Osawa, Hachioji, Tokyo 192-0397, Japan. E-mail: ffujibe.bs@gmail.com.

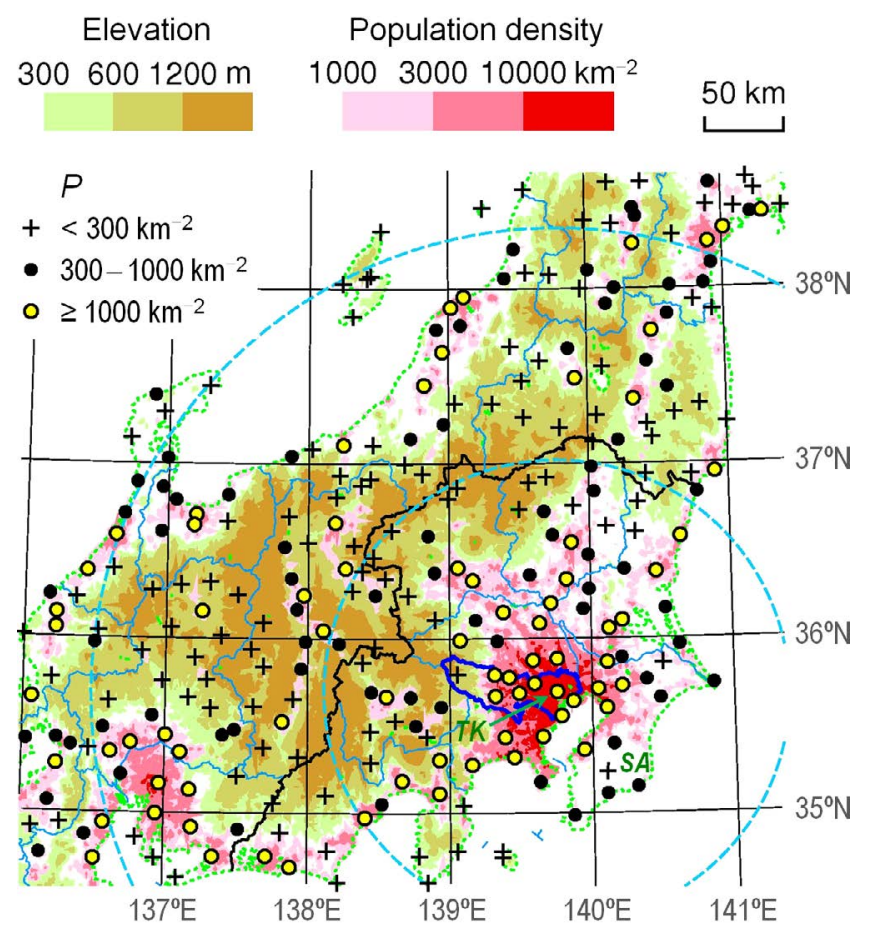

Fig. 1. Topography, population distribution, and AMeDAS stations in the area around Tokyo. Stations are shown in different symbols according to $P$, namely, population density near the site calculated from Eq.(1). Prefecture boundaries are shown in light blue lines, except for thick blue lines indicating the boundary of Tokyo Metropolis, and black lines for the service area of TEPCO. Dotted lines in light blue indicate $150 \mathrm{~km}$ and $300 \mathrm{~km}$ circles centered on the Tokyo station.

Area) comprised by 23 special wards with a total population of 9.27 million. The annual mean temperature at the JMA observatory in the central part of the Tokyo Ward Area is found to have risen by $3^{\circ} \mathrm{C}$ since the early 20th century (Matsumoto et al. 2017). Urban area has spread over surrounding prefectures to form the "Tokyo Metropolitan area" extending for several tens of kilometers, where a well-defined heat island is formed (Yamato et al. 2011; JMA 2018). The aim of this study is to find how the heat island in the Tokyo Metropolitan area was modified in the COVID-19 self-restraint period.

\section{Outline of the COVID-19 self-restraint in Japan}

Figure $2 \mathrm{a}$ shows the time series of daily new cases of COVID-19 infection in Japan (J.A.G Japan 2020). Infection was first reported on 16 January, and slowly spread in February. A moderate self-restraint began as the Prime Minister requested cancellation, delay, or scale-back of large events on 26 February. The self-restraint slightly relaxed in the middle of March, but again intensified as infection rapidly increased by the end of March. The Government declared a state of emergency to Tokyo Metropolis and twelve prefectures on 7 April, and extended it to the entire Japan on 17 April, so that a strong self-restraint continued until 
(a)
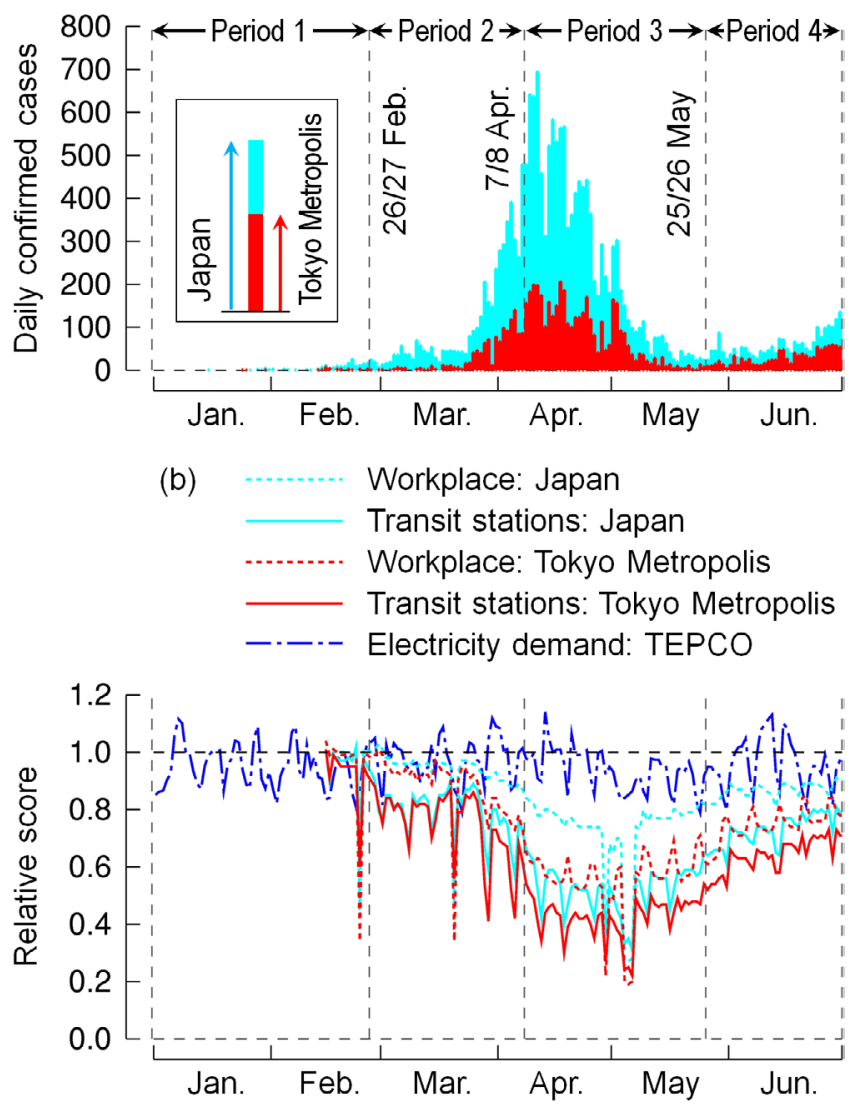

Fig. 2. Time series of (a) daily new cases of COVID-19 infection (J.A.G Japan 2020), and (b) electricity demand (TEPCO 2020; relative to 20172019 values) and movement trends in Google's Community Mobility Reports (Google LLC 2020; relative to 3 Jan. to 6 Feb. values).

the state of emergency was lifted from 14 to 25 May. After that human activity gradually restored.

In the present study, the former half of the year 2020 was divided into four periods according to the degree of self-restraint as

Period 1 (almost no restraint): 1 January to 26 February

Period 2 (moderate restraint): 27 February to 7 April

Period 3 (strong restraint): 8 April to 25 May

Period 4 (moderate to weak restraint): 26 May to 30 June

Figure $2 \mathrm{~b}$ shows the time series of some indices concerning human activities. The electricity demand to the Tokyo Electric Power Company (TEPCO 2020), which supplies electricity to Tokyo Metropolis and surrounding eight prefectures with a total population of 47.5 million (Fig. 1), changed only a little during the self-restraint period probably because of continued public services (e.g., railroads) and household demands. However, Google's Community Mobility Reports (Google LLC 2020) show a depression in movement trends from April to May, down to about 0.5 in transit stations and 0.7 in workplaces on the average over Japan. The depression was greater in Tokyo Metropolis, where movement trends were about 0.4 in transit stations and 0.5 in workplaces from April to May. It is therefore possible that anthropogenic heat release was so largely reduced in this period that could affect observed temperature. Details of the data shown in Fig. 2 are described in Supplement 1.

\section{Data and procedure of analysis}

\subsection{Data and selection of stations}

The study was made using hourly data on the network of the Automated Meteorological Data Acquisition System (AMeDAS) of JMA, including the Tokyo station in the central part of the Tokyo Ward Area (TK in Fig. 1). The site of the Tokyo station moved in December 2014 from a business area (Otemachi) to a park (Kitanomaru), resulting in a decrease of observed temperature by $0.9^{\circ} \mathrm{C}$ on the annual average (JMA 2016). The study is therefore based on data from 2015, up to June 2020.

The criteria of selecting stations for analysis are mostly the same as those used by Fujibe (2010). Data from stations at which the percentage of missing values exceeded $1 \%$ in any one of the six months from January to June were not used. To avoid the effects of discontinuities caused by site changes, data from stations that moved a horizontal distance of $1 \mathrm{~km}$ or more, or whose elevation changed by $5 \mathrm{~m}$ or more, were not used. Because a daily business cycle starts in the morning rather than at midnight, a day was defined as starting at 0600 Japan Standard Time (JST).

In order to select non-urban stations used for reference, population distribution data were obtained from the census results in 2015 for grid cells 30" of latitude and 45" of longitude in size (about $1 \mathrm{~km} \times 1 \mathrm{~km}$ ), available from the Portal Site of Official Statistics of Japan (e-Stat; https://www.e-stat.go.jp/gis/statmapsearch?page $=1 \&$ type $=1 \&$ toukeiCode $=00200521$ ) . The population density around station $i$ was calculated as a weighted average defined by

$$
P_{R}(i)=\frac{\sum_{g} \exp \left\{-\left(\frac{r_{i g}}{R}\right)^{2}\right\} P(g)}{\pi R^{2}},
$$

where $P(g)$ is the population of grid cell $g, r_{i g}$ is the distance of the grid cell from station $i$, and $R$ is a parameter indicating the size of the area in which the population density is to be calculated (Fujibe $2009,2010)$. In the present study $R$ was set to $3 \mathrm{~km}$. Stations at sites with $P<300 \mathrm{~km}^{-2}$ were regarded as non-urban and were used as reference stations for defining the urban anomaly, except for the Mt. Fuji station located at a height of $3775 \mathrm{~m}$. Although some urban temperature anomaly has been found even at stations with $P<300 \mathrm{~km}^{-2}$ (Fujibe 2009), these stations were regarded as nonurban and were used for reference in the present analysis, so that the spatial coverage of reference stations would be adequate. The subsequent analysis was based on data at 105 reference stations within $300 \mathrm{~km}$ of the Tokyo station. The reference station nearest to Tokyo is Sakahata (SA in Fig. 1), which is $60 \mathrm{~km}$ southeast of the Tokyo station.

\subsection{Definition of temperature anomaly in the self-restraint period}

The basic idea of the analysis is to compare the temperature at the Tokyo station observed in the self-restraint period with the temperature that would have been observed in a state without restraint. The latter was estimated from temperatures at reference stations on the basis of a regression equation that was developed using data for 2015 to 2019. The procedure is described in the following.

[1] For each station, the climate mean temperature was calculated for each time of the day on each date of the year. The calculation was done in the same way as that of JMA (2011) to produce daily climate normals, except that the data period was from 2015 to June 2020 in the present study. The subsequent analysis was based on temperature departure from the climate mean. It is denoted by $\Delta T_{i j}$, where $i$ is station and $j$ is date. In order to reduce short-term temperature fluctuation, a three-hour average temperature was used in a way that averages for 0100 to 0300 JST, 0400 to $0600 \mathrm{JST}, \ldots$, were regarded as the temperature at $0200 \mathrm{JST}$, $0500 \mathrm{JST}, \ldots$, respectively. Additionally, averages over $0700 \mathrm{JST}$ to $0600 \mathrm{JST}$ of the next day, $0700 \mathrm{JST}$ to $1800 \mathrm{JST}$, and $1900 \mathrm{JST}$ to 0600 JST of the next day were used as the daily mean, daytime, and nighttime temperatures, respectively.

[2] In order to express representative temperature patterns among the reference stations, principal component analysis was applied in the form 


$$
\Delta T_{i j}=\sum_{k=1}^{K} f_{i k} a_{i k}+\text { higher terms, }
$$

where $a_{i k}$ and $f_{j k}$ represent the spatial pattern and temporal change of the $k$-th principal component, respectively, and $K$ is the number of principal components used for the analysis. Since temperature patterns can vary according to time of the day and season, principal components were defined separately for each time of the day and each month. The analysis was based on a covariance matrix that was calculated with a weight $\exp \left[-\left(r / r_{0}\right)^{2}\right]$, where $r$ is the distance between the Tokyo station and a reference station, and $r_{0}=$ $150 \mathrm{~km}$. The value of $150 \mathrm{~km}$ for $r_{0}$ was given so that the temperature pattern influenced by the regional topography was captured. In the following analysis $K$ was set to 10 . The contribution of the ten components was $94.7 \%$ on the average over times of the day and months, with a minimum and maximum of $92.1 \%$ and $97.4 \%$, respectively.

[3] A regression equation for $\Delta T_{j}$ at Tokyo on date $j$, hereafter denoted by $\Delta T_{j}^{(\mathrm{TK})}$, was created from data for 2015 to 2019 using a least-squares condition

$$
\left[\Delta T_{j}^{(\mathrm{TK})}-\left(p_{0}+\sum_{k=1}^{K} p_{k} f_{j k}\right)\right]^{2} \rightarrow \text { minimum, }
$$

where $p_{k}(k=0, \ldots, K)$ is a least-squares coefficient. The regression was made for each month and time of the day for the same reason as in the step [2].

[4] The regression equation obtained in the step [3] was applied to the data in 2020 to obtain the temperature that would have been observed at Tokyo without restraint, denoted by $\Delta \widehat{T}_{j}^{(\mathrm{TK})}$. Then the anomaly of the observed temperature $\Delta T_{j}^{(\mathrm{TK})}$ was obtained as

$$
\delta \Delta T_{j}^{(\mathrm{TK})}=\Delta T_{j}^{(\mathrm{TK})}-\Delta \hat{T}_{j}^{(\mathrm{TK})} .
$$

[5] The value of $\delta \Delta T_{j}^{(\mathrm{TK})}$ on each day was averaged over each of the four periods defined in Section 2. The averaged value of $\delta \Delta T_{j}^{(\mathrm{TK})}$ will be denoted by $\left\langle\delta \Delta T^{(\mathrm{TK})}\right\rangle$. It is to be noted that $\left\langle\delta \Delta T^{(\mathrm{TK})}\right\rangle$ is a function of time of the day. The confidence range of $\left\langle\delta \Delta T^{(\mathrm{TK})}\right\rangle$ was based on the $t$-distribution. The validity of applying the $t$-test is discussed in Supplement 2.

The above-mentioned analysis has a number of options in procedure and parameters such as $R, r_{0}$, and $K$. In order to check the sensitivity of the result to these options, a series of comparative analyses based on different options was made. The results are shown in Supplement 3. Additionally, the procedure from the steps [3] to [5] was applied also to other stations in the vicinity of Tokyo in order to obtain the spatial pattern of $\langle\delta \Delta T\rangle$.

\section{Results}

Figure 3a shows the time series of $\delta \Delta T_{j}^{(\mathrm{TK})}$ for daily mean temperature. The values of $\delta \Delta T_{j}^{(\mathrm{TK})}$ tends to be negative from April to May, although there are large day-to-day fluctuations. Figure $3 \mathrm{~b}$ shows the diurnal variation of $\left\langle\delta \Delta T^{(\mathrm{TK})}\right\rangle$ for each (a)
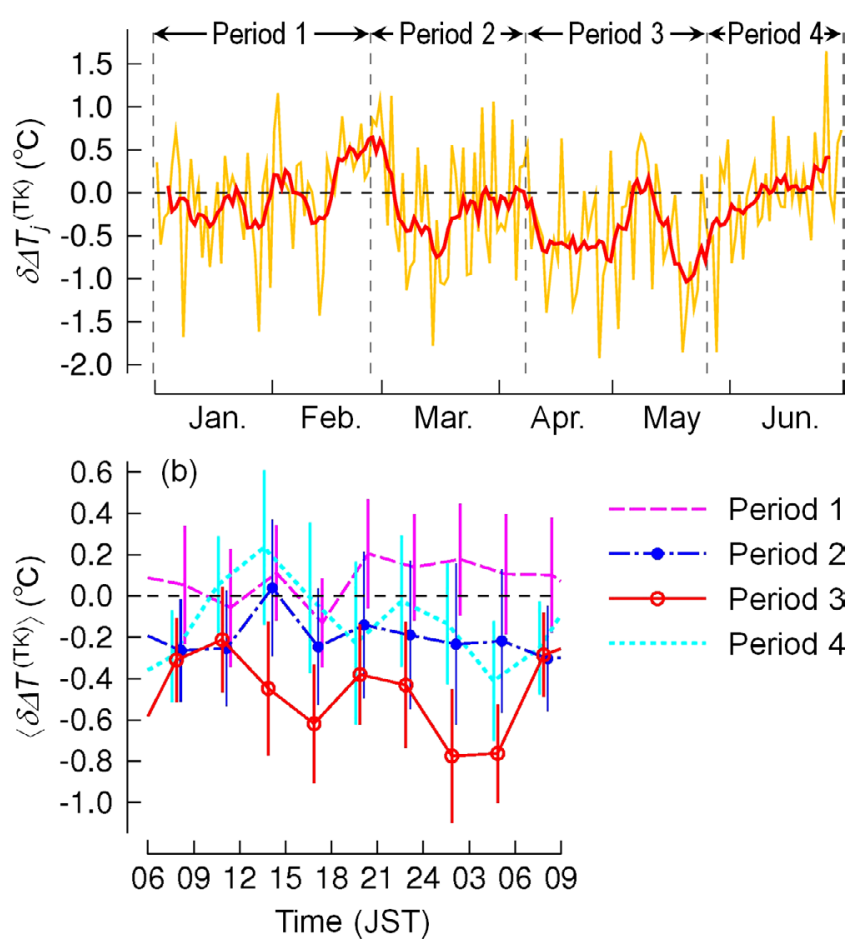

Fig. 3. (a) Time series of $\delta \Delta T_{j}^{(\mathrm{TK})}$ defined by Eq.(4), and its seven-day running mean. (b) Diurnal variation of $\left\langle\delta \Delta T^{(\mathrm{TK})}\right\rangle$ for each period, with side shift in order to avoid overlapping. Vertical bars in (b) indicate the range of $95 \%$ confidence.

period. The values of $\left\langle\delta \Delta T^{(\mathrm{TK})}\right\rangle$ for the daily mean, daytime, and nighttime are shown in Table 1. For Period $1,\left\langle\delta \Delta T^{(\mathrm{TK})}\right\rangle$ is statistically insignificant all day, while that in Period 2 has negative values of about $-0.2^{\circ} \mathrm{C}$ in the morning and evening, and is significant at the $10 \%$ level for the daily mean and the daytime. For Period 3, $\left\langle\delta \Delta T^{(\mathrm{TK})}\right\rangle$ has negative values exceeding $-0.5^{\circ} \mathrm{C}$ in the evening and early morning, with a significance of the $1 \%$ level. In Period $4,\left\langle\delta \Delta T^{(\mathrm{TK})}\right\rangle$ is small and insignificant on the daily mean, although the value at $0500 \mathrm{JST}\left(-0.41^{\circ} \mathrm{C}\right)$ is significant at the $1 \%$ level.

Figure 4 shows the distribution of $\left\langle\delta \Delta T^{(\mathrm{TK})}\right\rangle$ in Period 3 for stations in the area around Tokyo. For daily mean temperature, many stations surrounding Tokyo have negative $\left\langle\delta \Delta T^{(\mathrm{TK})}\right\rangle$ values that tend to diminish with the distance from Tokyo, except for a few stations having large absolute values of $\left\langle\delta \Delta T^{(\mathrm{TK})}\right\rangle$. The situation is basically the same for the daytime and the nighttime, although $\left\langle\delta \Delta T^{(\mathrm{TK})}\right\rangle$ is larger in the nighttime than in the daytime. Figure 5 shows the diurnal variation of $\left\langle\delta \Delta T^{(\mathrm{TK})}\right\rangle$ for Period 3 averaged over stations in each circular area having a $25 \mathrm{~km}$ width,

\begin{tabular}{|c|c|c|c|c|}
\hline Period & Station and area & Daily mean (07-06 JST) & Daytime (07-18 JST) & Nighttime (19-06 JST) \\
\hline 2 & Tokyo (Kitanomaru) & $\underline{-0.19} \pm 0.20$ & $\underline{-0.18} \pm 0.19$ & $-0.20 \pm 0.30$ \\
\hline 4 & Tokyo (Kitanomaru) & $-0.10 \pm 0.20$ & $-0.00 \pm 0.23$ & $-0.20 \pm 0.27$ \\
\hline
\end{tabular}

Table 1. List of $\left\langle\delta \Delta T^{(\mathrm{TK})}\right\rangle$ at Tokyo for each period, and for each circular area in Period 3. Units are in ${ }^{\circ} \mathrm{C}$, and a plus-minus sign indicates the $95 \%$ confidence range.

Underlines indicate significance at the $\underline{\underline{1 \%}}$ and the $\underline{10 \%}$ levels. 


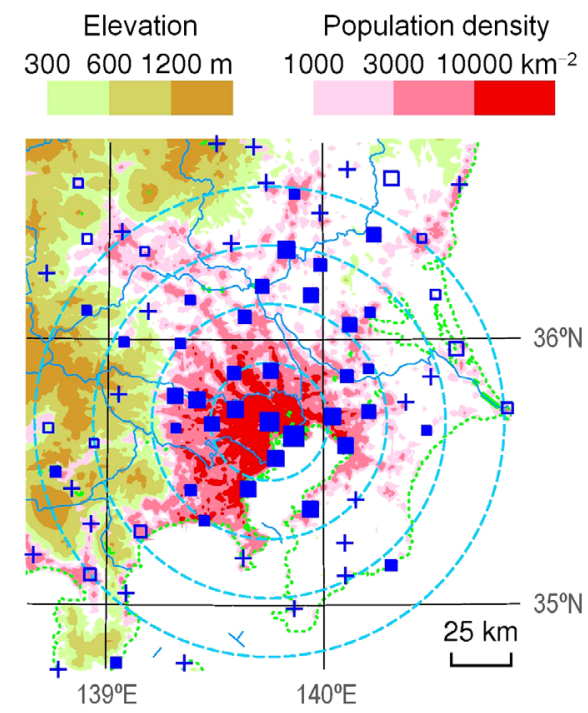

(a) Daily mean

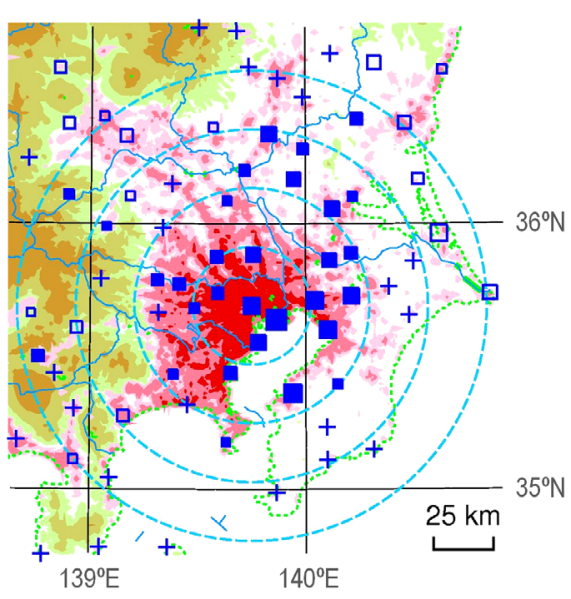

(b) Daytime
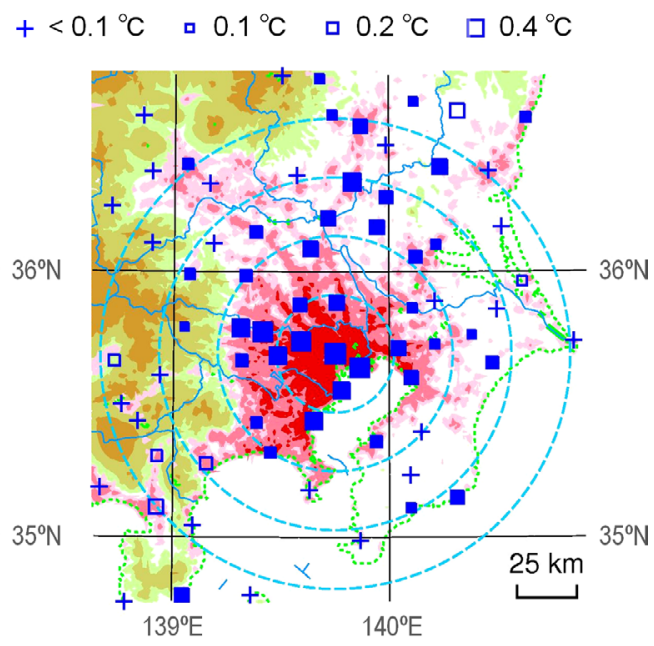

(c) Nighttime

Fig. 4. Distribution of $\langle\delta \Delta T\rangle$ at stations near Tokyo in Period 3. Open and filled squares indicate positive and negative values, respectively, and their areas are proportional to the absolute values of $\langle\delta \Delta T\rangle$. Crosses indicate stations where the absolute value of $\langle\delta \Delta T\rangle$ is smaller than $0.1^{\circ} \mathrm{C}$. Dotted light blue lines indicate $25 \mathrm{~km}, 50 \mathrm{~km}, 75 \mathrm{~km}$, and $100 \mathrm{~km}$ circles centered on the Tokyo station.

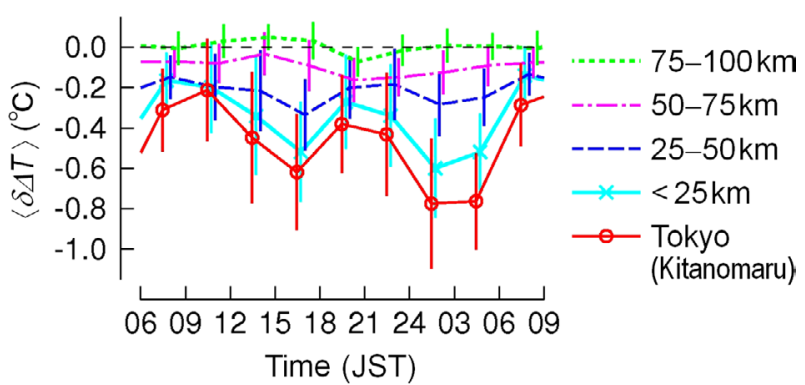

Fig. 5. Diurnal variation of $\langle\delta \Delta T\rangle$ in Period 3 for the Tokyo station and the average over stations in each range from the Tokyo station. The way of presentation is the same as that in Fig. $3 \mathrm{~b}$.

with the Tokyo station as the center. The values of $\left\langle\delta \Delta T^{(\mathrm{TK})}\right\rangle$ for the daily mean, daytime, and nighttime are shown in Table 1. Although $\left\langle\delta \Delta T^{(\mathrm{TK})}\right\rangle$ decreases with distance from Tokyo, statistically significant negative values are found as far as in the 50$75 \mathrm{~km}$ area. Thus the negative temperature anomaly in Period 3 is a feature extending for several tens of kilometers from the center of Tokyo.

Finally, the same procedure as above was applied to six cities that have a population over a million and are in the central part of other regions of Japan. Figure 6 shows the location of these cities, and the diurnal variation of $\langle\delta \Delta T\rangle$ in Period 3 at the station in each city. Sendai and Fukuoka show negative $\langle\delta \Delta T\rangle$ with daily mean values of $-0.26^{\circ} \mathrm{C}$ and $-0.17^{\circ} \mathrm{C}$, respectively. These values are statistically significant at the $1 \%$ level. However, $\langle\delta \Delta T\rangle$ in other cities is insignificant, although $\langle\delta \Delta T\rangle$ has more negative values than positive values.

\section{Summary and remarks}

The present study has revealed negative temperature anomaly in the Tokyo Metropolitan area in the depth of COVID-19 self-restraint from April to May 2020 (Period 3). The temperature anomaly in central Tokyo is about $-0.5^{\circ} \mathrm{C}$ on the daily mean, and is larger in the nighttime than in the daytime. Negative temperature anomaly is found in an area extending for several tens of ki- (a)
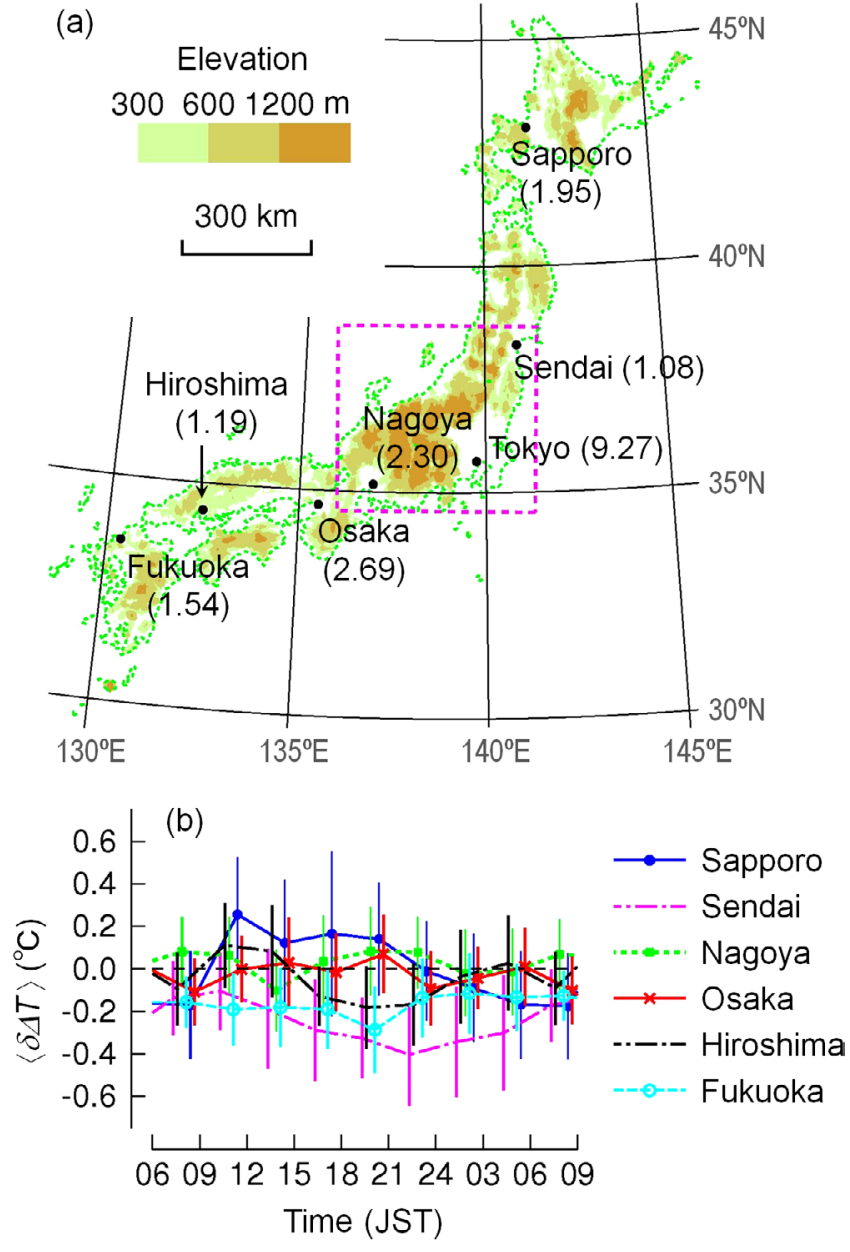

Fig. 6. (a) Location of cities used for the analysis in (b), together with the Tokyo Ward Area. Parentheses indicate the population of cities and the Tokyo Ward Area. (b) Diurnal variation of $\langle\delta \Delta T\rangle$ in Period 3 for the six cities. The way of presentation is the same as that in Fig. $3 \mathrm{~b}$. 
lometers from Tokyo. In central Tokyo, a weak negative anomaly of about $-0.2^{\circ} \mathrm{C}$ is found also in the period of weak self-restraint from the end of February to the beginning of April (Period 2).

The net warming in central Tokyo since the early 20th century has been evaluated to be $3^{\circ} \mathrm{C}$ (Matsumoto et al. 2017), implying urban contribution of about $2^{\circ} \mathrm{C}$ in addition to the large-scale warming of about $1{ }^{\circ} \mathrm{C}$. Thus the depression of $-0.5^{\circ} \mathrm{C}$ in the self-restraint period accounts for a substantial fraction of the total urban warming of Tokyo. This result is qualitatively consistent with the results of simulation studies that showed considerable contribution of anthropogenic heat to the formation of urban heat island, especially in the nighttime (Ichinose et al. 1999; Kusaka and Kimura 2004; Ryu and Baik 2012; Aoyagi et al. 2012; Li et al. 2013). The larger temperature anomaly in comparison to the weekday-holiday temperature difference (about $0.2^{\circ} \mathrm{C}$; Fujibe 1988, 2010) implies a stronger suppression of human activity with a larger depression of anthropogenic heat release during the self-restraint period than on normal holidays, in agreement with our impression at that time. However, quantitative discussion will require an evaluation of the amount of anthropogenic heat emission in the self-restraint period, as well as detailed inspection of the results of numerical simulations that show some diversity in the intensity of the effect of anthropogenic heat on temperature.

The widespread temperature anomaly in the self-restraint period (Fig. 4) is similar to the feature of temperature anomaly on holidays (Fujibe 1988), with respect to an area of negative anomaly covering a large area in and around Tokyo. These results are consistent with a widespread heat island obtained from simulations (e.g., JMA 2018). However, a report on detailed distribution of anthropogenic heat shows an area of high emission limited to the central part of the Tokyo Ward Area (Ministry of Land, Infrastructure, Transport and Tourism and Ministry of the Environment 2004; Aoyagi et al. 2012). Further studies will be needed to make clear the horizontal scale of the area affected by anthropogenic heat.

The present study has indicated a possibility of temperature decrease in the self-restraint period in some other large cities as well (Fig. 6). However, an accurate evaluation of temperature anomaly has been hindered by large statistical uncertainty. The absence of significant anomaly for Osaka, which is the central city of the second largest urban area in Japan having a population of more than ten million, may appear to be inconsistent with the large anomaly for Tokyo. However, this can be a result of statistical fluctuation that has concealed the signal. It is a future task to make a more accurate evaluation of temperature anomaly for Osaka and other large cities.

\section{Supplements}

Supplement 1: Details of time series data used for Fig. 2. aly.

Supplement 2: Applicability of the t-test to temperature anom-

Supplement 3: Comparison of results from different options in the analysis.

\section{References}

Aoyagi, T., N. Kayaba, and N. Seino, 2012: Numerical simulation of the surface air temperature change caused by increases of urban area, anthropogenic heat, and building aspect ratio in the Kanto-Koshin area. J. Meteor. Soc. Japan, 90B, 11-31.

Dou, J., and S. Miao, 2017: Impact of mass human migration during Chinese New Year on Beijing urban heat island. Int. J. Climatol., 37, 4199-4210.

Earl, N., I. Simmonds, and N. Tapper, 2016: Weekly cycles in peak time temperatures and urban heat island intensity. Environ.
Res. Lett., 11, 074003, doi:10.1088/1748-9326/11/7/074003.

Fujibe, F., 1988: Weekday-weekend differences of urban climates. Part 3: Temperature and wind fields around Tokyo and Osaka. J. Meteor. Soc. Japan, 66, 377-385.

Fujibe, F., 2009: Detection of urban warming in recent temperature trends in Japan. Int. J. Climatol., 29, 1811-1822.

Fujibe, F., 2010: Day-of-the-week variations of urban temperature and their long-term trends in Japan. Theor. Appl. Climatol., 102, 393-401.

Google LLC, 2020: Google COVID-19 Community Mobility Reports. (Available online at https://www.google.com/ covid19/mobility/, accessed 7 July 2020).

Ichinose, T., K. Shimodozono, and K. Hanaki, 1999: Impact of anthropogenic heat on urban climate in Tokyo. Atmos. Environ., 33, 3897-3909.

J. A. G Japan, 2020: Documents of the Data "Dashboard of Coronavirus COVID-19 Japan Case (2019-nCoV)". (Available online at https://jag-japan.com/covid19map-readme/, accessed 7 July 2020). (in Japanese)

Japan Meteorological Agency (Observations Department), 2011: On the method of producing the 2010 climate normals. Wea. Serv. Bull., 78, 43-56. (in Japanese)

Japan Meteorological Agency (Observations Department and Tokyo Regional Headquarters), 2016: On the site change of the Tokyo surface observation station. Wea. Serv. Bull., 83, 1-32. (in Japanese)

Japan Meteorological Agency, 2018: Heat Island Monitoring Report 2017. 66 pp. (Available online at https://www.data. jma.go.jp/cpdinfo/himr/h30/index.html, accessed 7 July 2020). (in Japanese)

Kusaka, H., and F. Kimura, 2004: Thermal effects of urban canyon structure on the nocturnal heat island: Numerical experiment using a mesoscale model coupled with an urban canopy model. J. Appl. Meteor. Climatol., 43, 1899-1910.

Li, X.-X., T.-Y. Koh, D. Entekhabi, M. Roth, J. Panda, and L. K. Norford, 2013: A multi-resolution ensemble study of a tropical urban environment and its interactions with the background regional atmosphere. J. Geophys. Res. Atmos., 118, 9804-9818.

Matsumoto, J., F. Fujibe, and H. Takahashi, 2017: Urban climate in the Tokyo metropolitan area in Japan. J. Environ. Sci., 59, 54-62.

Ministry of Land, Infrastructure, Transport and Tourism, and Ministry of the Environment, 2004: Report on Heat Island Countermeasures by Controlling Anthropogenic Heat in Cities. Ministry of the Environment, $165 \mathrm{pp}$. (Available online at https://www.env.go.jp/air/report/h16-05/, accessed 7 July 2020). (in Japanese)

Ryu, Y.-H., and J.-J. Baik, 2012: Quantitative analysis of factors contributing to urban heat island intensity. J. Appl. Meteor. Climatol., 51, 842-854.

Tokyo Electric Power Company, 2020: Download Past Electricity Demand Data. (Available online at https://www.tepco.co.jp/ en/forecast/html/download-e.html, accessed 7 July 2020).

Yamato, H., T. Mikami, and H. Takahashi, 2011: Influence of sea breeze on the daytime urban heat island in summer in the Tokyo Metropolitan Area. J. Geogr., 120, 325-340. (in Japanese with English abstract)

Zhang, J., and L. Wu, 2017: Influence of human population movements on urban climate of Beijing during the Chinese New Year holiday. Sci. Rep., 7, 45813, doi: 10.1038/srep45813.

Zhang, J., L. Wu, F. Yuan, J. Dou, and S. Miao, 2015: Mass human migration and Beijing's urban heat island during the Chinese New Year holiday. Sci. Bull., 60, 1038-1041.

Manuscript received 10 July 2020, accepted 14 August 2020

SOLA: https://www.jstage.jst.go.jp/browse/solal 